\title{
L'hétérochromatine constitutive dans tous ses états
}

> Les centromères, lieu d'assemblage des kiné-

Rémi Terranova

tochores, sont cruciaux pour la ségrégation des chromosomes lors de la mitose et de la méiose. Leur altération peut conduire à l'aneuploïdie, une anomalie génétique très fréquemment observée dans les tumeurs humaines. Ces régions sont organisées sous forme d'hétérochromatine dite «constitutive», et restent condensées tout au long du cycle cellulaire. L'hétérochromatine constitutive a longtemps été perçue comme une structure stable et inerte. Cette conception a depuis largement évolué, de nombreuses études montrant sa plasticité et lui associant de multiples fonctions dans les cellules en interphase. Cet article présente certains aspects de cette plasticité de l'hétérochromatine constitutive et souligne l'importance de l'intégrité épigénétique des centromères pour la stabilité du génome. <

Plusieurs niveaux de contrôles épigénétiques sont impliqués dans la régulation du génome. Des modifications locales de la chromatine (comme les modifications des histones) permettent le recrutement (ou l'exclusion) de complexes multiprotéiques (comme les protéines des groupes Polycomb ou Trithorax). Ces complexes protéiques organisent la chromatine localement pour la régulation fonctionnelle du génome. Des composants ribonucléiques sous la forme d'ARN non-codants jouent aussi un rôle dans la régulation de l'expression et l'organisation structurale du génome. Ces multiples et complexes niveaux d'organisation du génome définissent différentes formes de chromatines, avec des propriétés physiques et biochimiques distinctes. Au niveau cytologique, les observations de Heitz en 1928 [1] ont permis de distinguer différents états de condensation de la chromatine chez les eucaryotes. L'euchromatine d'une part, riche en gènes, est organisée de façon différentielle dans le temps et l'espace, en fonction de l'état transcriptionnel. Dans leur forme inactive, ces régions peuvent être physiquement condensées, en association avec des marques épigénétiques répressives et donner naissance à l'hétérochromatine dite facultative. L'hé-

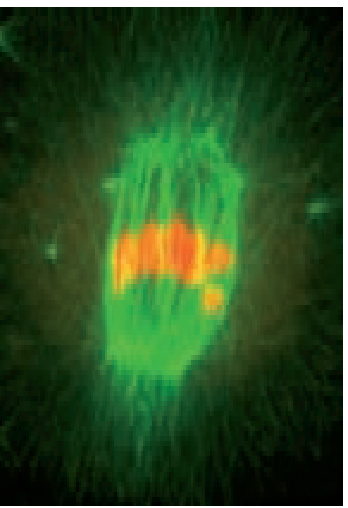

Friedrich Miescher Institute (FMI), Novartis Research Foundation, Maulbeerstrasse 66, CH-4058, Basel, Suisse. remi.terranova@fmi.ch

térochromatine constitutive d'autre part, pauvre en gènes, contient une grande quantité d'ADN hautement répétitif. Ces régions sont condensées et visibles tout au long du cycle cellulaire. Dans la plupart des organismes, l'hétérochromatine constitutive est présente autour des centromères et près des régions télomériques des chromosomes. Ces régions sont importantes pour la stabilité du génome; les centromères en particulier permettent l'organisation du kinétochore et la ségrégation des chromosomes lors de la mitose et de la méiose.

\section{Modifications épigénétiques des centromères chez la souris}

Chez la souris, les centromères sont formés de répétitions en tandem de séquences «satellites mineures» (régions centriques), lieu d'assemblage du kinétochore, flanquées de séquences «satellites majeures 》 (régions péricentriques) (Figure 1A). Dans le noyau en interphase, ces régions sont condensées et se distinguent par une configuration épigénétique qui leur est propre (pour revues, voir [2, 3, 37]). Ces caractéristiques épigénétiques sont essentielles pour établir et maintenir l'identité d'un centromère fonctionnel.

Ainsi, la structuration même des nucléosomes et la présence de variants d'histones distinguent les régions centromériques du reste du génome. En particulier, au niveau du centromère, la protéine CENP-A (CENtromere protein $A$ ) remplace l'histone $\mathrm{H} 3$ (Figure IB). La présence de CENP-A est essentielle à l'organisation du kinétochore et à la ségrégation des chromosomes. 
Un variant pour l'histone H2A (H2A.Z) a aussi été détecté le long des régions centriques et péricentriques. Ces régions sont par ailleurs caractérisées par un faible degré d'acétylation des histones et une tri-méthylation fréquente de la lysine 9 de l'histone H3 (H3K9me3), une marque établie par les histone-méthyltransférases (HMT) Suv39hl et h2. Les différentes isoformes de la protéine HPl $(\alpha, \beta$ et $\gamma)$, probablement recrutées par $\mathrm{H} 3 \mathrm{~K} 9 \mathrm{me} 3$, sont, elles, enrichies spécifiquement au niveau des régions péricentriques et pourraient, via une interaction avec Suv39h, permettre le maintien dynamique et la propagation de cet état épigénétique [39]. Les centromères et régions péricentriques, enfin, sont enrichis en H3K27mel et H4K20me3 et sont méthylés au niveau des $C p G$ via le recrutement des ADN méthyltransférases Dnmt3a et $3 b$. Outre la nature et l'organisation linéaire des répétitions en tandem et la présence de CENP-A, les centromères se distinguent épigénétiquement des régions péricentriques par la présence de H3K4me2, une marque associée à l'activité transcriptionnelle, et par l'absence des protéines $\mathrm{HPl}$. Alors que CENP-A et la structuration épigénétique des centromères sont conservées au cours de l'évolution, les séquences centriques et péricentriques sous-jacentes le sont peu et sont finalement secondaires dans la définition d'un centromère fonctionnel (pour revue, voir [4]).

\section{Dynamique transcriptionnelle de l'hétérochromatine}

Loin d'être transcriptionnellement inactives, les régions centromériques produisent de longs ARN non-codants (pour revue, [5]). Chez la levure S. pombe, ces ARN centromériques sont nécessaires à l'établissement et au maintien de la configuration épigénétique et par conséquent à l'intégrité des centromères. En particulier, le ciblage aux centromères de l'HMT Clr4, homologue de Suv39h, et donc de la méthylation de $\mathrm{H} 3 \mathrm{~K}$ 9, dépend de la machinerie d'inactivation par l'ARN (ARNi) et de la formation de petits ARN interférents (siARN) issus des régions centromériques $[6,7]$. L'assemblage de CENP-A, si important pour la formation du kinétochore, dépend aussi de ces siARN [8]. Ces répétitions centromériques sont exprimées transitoirement au cours de la phase $S$, suggérant une régulation dynamique, dépendante du cycle cellulaire, des niveaux d'expression des ARN non-codants [9]. Les centromères chez les eucaryotes supérieurs produisent aussi des ARN non-codants (Figure 1A) [10-12] qui ne sont apparemment pas associés à la production de siARN. Pourtant, la machinerie d'interférence elle-même semble jouer un rôle dans la structuration des centromères puisque l'inactivation de la RNAse III dicer conduit à des altérations de l'architecture hétérochromatinienne centromérique [13]. Par ailleurs, des expériences de traitement de cellules vivantes par la RNase ont montré qu'un composant ribonucléique est nécessaire au maintien de l'organisation des centromères et à la présence de HPl à l'hétérochromatine péricentrique [14], mais la nature de l'ARN mis en jeu reste inconnue. Chez le maïs, des ARN d'origine centromérique font partie intégrante du kinétochore [12]. Ces résultats soulignent la dynamique transcriptionnelle des régions centriques et péricentriques. Bien que potentiellement conservé, le rôle exact des transcrits centromériques et de la machinerie ARNi dans l'assemblage, le maintien et la fonction des centromères reste à établir chez les eucaryotes supérieurs.

\section{Plasticité structurale et fonctionnelle de l'hétérochromatine}

Comme son nom l'indique, l'hétérochromatine constitutive a longtemps été perçue comme inerte et de composition invariable. Une très large littérature ces dernières années a contribué à changer cette perception en montrant sa plasticité et en lui associant de multiples fonctions dans les cellules en interphase.

L'examen microscopique détecte facilement les régions péricentriques des noyaux de cellules murines, riches en $A / T$, après marquage au DAPI (4',6-diamidino-2-phenylindole) et permet de distinguer l'hétérochromatine constitutive de l'euchromatine. L'analyse de l'organisation tridimensionnelle (3D) de l'hétérochromatine constitutive a démontré son extraordinaire plasticité spatiale. Un des exemples les plus frappants de plasticité structurale et épigénétique des domaines (péri)-centromériques a récemment été décrit au cours du développement embryonnaire précoce chez la Souris. Dans les zygotes, les génomes parentaux sont épigénétiquement asymétriques $[15,41](\rightarrow)$.

Dans ces cellules, l'hétérochromatine est organisée en anneaux autour des précurseurs des nucléoles [16, $17]$; alors que l'hétérochromatine maternelle est associée avec les marques épigénétiques conventionnelles (H3K9me2/3 et HP1 $\beta$ ), la plupart de ces marques sont absentes au niveau de l'hétérochromatine paternelle (pour revue, voir [3]). Étonnamment, elles y sont remplacées par d'autres modifications épigénétiques dont H3K27me3, et la présence du complexe de répression PRCl (Polycomb repressive complex 1) [17]. Par ailleurs, dans les cellules souches embryonnaires déficientes pour les HMT Suv39h1/h2, la marque H3K27me3, établie par la protéine PcG Ezh2 et caractéristique de I'hétérochromatine facultative, est aussi enrichie au niveau de l'hétérochromatine constitutive [18]. Les protéines PcG sont normalement exclues de l'hétérochromatine constitutive et plutôt associées à la régulation des régions euchromatiques via la formation de I'hétérochromatine facultative [38]. Ces observations suggèrent un rôle pour les protéines du groupe Polycomb dans la régulation de l'hétérochromatine constitutive en l'absence de $\mathrm{H} 3 \mathrm{~K} 9 \mathrm{me} 3$ et sont importantes pour la compréhension des modes de régulation et de propagation des patrons épigénétiques au niveau des régions (péri)-centromériques. 
Dans les noyaux en interphase, les centromères de plusieurs chromosomes se regroupent pour former des «chromocentres». Ces chromocentres sont visibles dans tous les types cellulaires, mais leur composition varie généralement d'un type cellulaire à un autre ainsi qu'au cours de la différenciation [19-21]. Ces changements peuvent être dépendants du cycle cellulaire [22, 23 ] et sont expérimentalement réversibles. Ainsi, des expériences de reprogrammation nucléaire par fusion cellulaire (hétérokaryons) et clonage ont montré la plasticité de l'organisation nucléaire en général et des centromères en particulier qui adoptent rapidement la configuration associée à leur nouvelle identité cellulaire $[24,25]$. L'organisation spatiale des régions hétérochromatiques dans le noyau pourrait jouer un rôle actif dans la différenciation cellulaire, en formant un sous compartiment nucléaire fonctionnel dédié à la répression de certains gènes. Plusieurs études ont ainsi montré le repositionnement nucléaire de locus dans des processus de différenciation associés à des changements d'expression des gènes. L'hétérochromatine péricentrique, en particulier, joue un rôle important pour l'inactivation du locus brown dans les embryons de drosophile [26] et pourrait être nécessaire au maintien de l'état transcriptionnellement inactif de certains gènes au cours de la différenciation lymphocytaire chez la souris (pour revues [27, 40]. Au-delà de son rôle dans la régulation stable et transmissible à la descendance des patrons d'expression de certains gènes, plusieurs études ont par ailleurs récemment démontré que l'hétérochromatine péri-centromérique pourrait être impliquée dans le phénomène d'exclusion allélique, responsable de l'expression mono-allélique des gènes d'immunoglobuline et par conséquent de la spécificité immunologique des lymphocytes B [28].

Dans leur ensemble, ces résultats illustrent la plasticité de l'organisation 3D et la multiplicité fonctionnelle des régions centromériques. Chez la levure S. pombe, I'hétérochromatine constitutive est considérée comme une plate-forme dynamique pour le recrutement et la propagation de nombreux régulateurs impliqués dans diverses voies de régulation (transcription, ségrégation des chromosomes, réplication) (pour revue, voir [29]). Il est tentant de spéculer que l'hétérochromatine constitutive chez les eucaryotes supérieurs constitue aussi une plate-forme d'assemblage associée à de nombreuses fonctions au cours du cycle cellulaire et de la différenciation cellulaire normale et pathologique.

\section{Hétérochromatine, stabilité du génome et cancer}

Le kinétochore est composé de nombreuses protéines, permettant l'interaction entre les chromosomes et le fuseau mitotique pour la ségrégation des chromatides sœurs dans les cellules filles au cours de la division cellulaire. L'altération d'un ou plusieurs composants du kinétochore conduit à des défauts de ségrégation des chromosomes et à l'aneuploïdie (nombre anormal de chromosomes), caractéristique des tumeurs solides chez l'homme [30]. Compte tenu du rôle des centromères dans l'organisation du kinétochore, leur intégrité structurale et épigénétique est essentielle au maintien de la ploïdie et à la stabilité du génome. Ainsi, la mutation/dérégulation de CENP-A (variant centromérique de l'histone $\mathrm{H} 3$ ) conduit à une perte totale de la ségrégation des chromosomes chez tous les eucaryotes. Theodore Boveri a

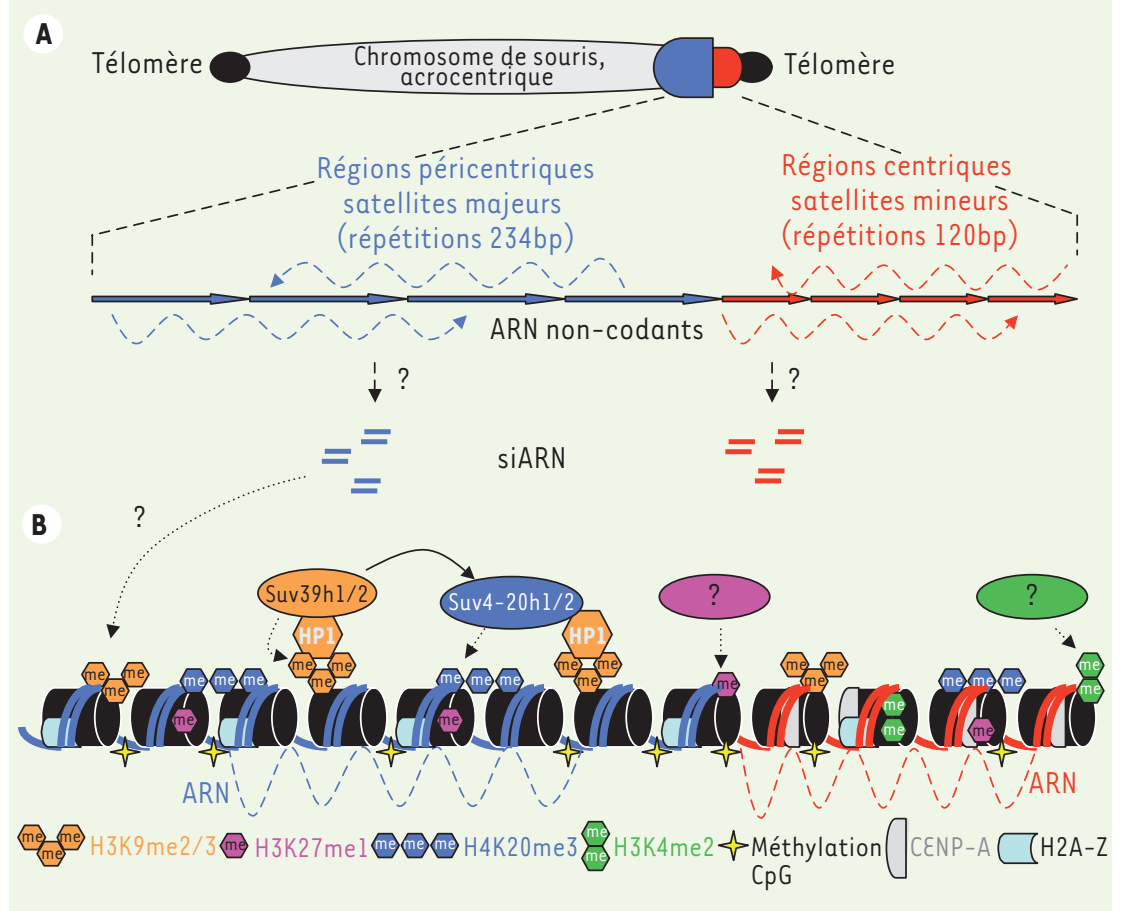

Figure 1. Bases (épi)génétiques des centromères chez la souris. A. Représentation schématique d'un chromosome de souris acrocentrique et de l'organisation en tandem des régions centriques (satellites mineurs) et péricentriques (satellites majeurs). Les flèches en pointillés indiquent la production de longs ARN non-codants. B. Organisation épigénétique des régions centriques et péricentriques chez la souris. Au niveau des régions centriques, lieu d'assemblage du kinétochore, I'histone $\mathrm{H} 3$ est remplacée par le variant CENP-A. Les HMT Suv39hl/h2 sont responsables de l'établissement et du maintien de l'hétérochromatine constitutive aux régions centriques et péricentriques. En plus de $\mathrm{H} 3 \mathrm{~K} 9 \mathrm{me} 3$, un constituant ribonucléique est nécessaire au maintien de HPI à l'hétérochromatine péricentrique [14] (modifié d'après [2]). 
proposé il y a un siècle un lien de cause à effet entre aneuploïdie et tumorigenèse [31]. Cependant, cette proposition n'a pu être testée que récemment par la génération de modèles murins montrant des taux élevés d'aneuploïdie en l'absence d'autres défauts apparents. C'est le cas dans les cellules et souris déficientes pour CENP-E (CENtromere protein $\varepsilon$ ), un autre composant centromérique important pour la ségrégation mitotique des chromosomes [32]. Des cellules et souris déficientes pour ce gène deviennent aneuploïdes et des lymphomes spontanés ainsi que des tumeurs du poumon apparaissent chez les animaux âgés. De nombreux autres constituants du kinétochore sont indispensables pour la ségrégation des chromosomes, et leur dérégulation conduit à l'instabilité des chromosomes [33]. Toutefois, un nombre croissant d'études montrent qu'au-delà du kinétochore, chaque composant de l'hétérochromatine constitutive aux (péri)-centromères (et probablement la stœchiométrie de ses composants majeurs, dont les ARN non-codants) est essentiel à l'assemblage et à la fonction du centromère/kinétochore [5]. Ainsi la perte de $\mathrm{H} 3 \mathrm{~K} 9 \mathrm{me} 3 \mathrm{au}$ niveau de l'hétérochromatine dans les souris déficientes pour les HMT Suv39h conduit à l'instabilité des chromosomes [34]. L'organisation de l'hétérochromatine constitutive et la ségrégation des chromosomes sont aussi altérées lors de changements des niveaux d'acétylation des histones après l'inhibition expérimentale des enzymes responsables de la désacétylation des histones (HDAC) [35]. Enfin, des changements du niveau d'expression des ARN centromériques conduisent à la désorganisation épigénétique des régions (péri)-centromériques associée à des défauts de ségrégation des chromosomes et de cohésion des chromatides sœurs [36]. Les recherches futures permettront peutêtre d'établir un lien mécanistique entre ces défauts d'organisation de l'hétérochromatine constitutive aux centromères et la cancérogenèse.

\section{Conclusion}

L'ensemble de ces résultats suggère que loin d'être une structure invariable, l'hétérochromatine est structuralement plastique et paradoxalement transcriptionnellement active. Elle varie d'un type cellulaire à un autre, au cours du cycle cellulaire ainsi qu'au cours du développement embryonnaire précoce. Outre leur rôle dans la ségrégation des chromosomes, les régions centromériques sont associées à la régulation de l'expression transcriptionnelle. La compréhension des bases génétiques et épigénétiques de la stabilité du génome, dont l'établissement et le maintien des régions (péri)-centromériques, permettra éventuellement de découvrir de nouvelles cibles thérapeutiques pour le traitement des cancers. L'étude de l'hétérochromatine constitutive, paradigme de régulation épigénétique, est par ailleurs importante pour la compréhension de voies de régulation épigénétiques applicables au reste du génome, dans un contexte sain et pathologique. $\diamond$

\section{SUMMARY}

The many faces of constitutive heterochromatin

Constitutive heterochromatin, key for the structuration of the centromeres, was long thought as a transcriptionally inert compartment, relatively invariant in composition and structure. Numerous studies have now challenged this view by showing the versatility of these epigenetically defined regions. This review highlights some important aspects of (peri)-centromeric heterochromatin structural and functional plasticity in development and differentiation. The integrity of centromeric regions is crucial for the stability of the genome and the maintenance of ploidy. I outline recent evidence suggesting that epigenetic alterations of heterochromatin can cause defects in chromosome segregation leading to aneuploidy and tumorigenesis. $\diamond$

\section{REMERCIEMENTS}

L'auteur remercie Véronique Azuara (IRDB, Londres) pour ses critiques et suggestions dans la préparation de cet article. Merci à l'Epigenome NoE, au Friedrich Miescher Institute (FMI) et au Laboratoire du Dr Antoine Peters pour leur soutien financier.

\section{RéFÉRENCES}

1. Heitz $\varepsilon$. Das heterochromatin der Moose. Jb Wiss Bot $1928 ; 69: 728$.

2. Peters $A H$, Schubeler D. Methylation of histones: playing memory with DNA. Curr Opin Cell Biol 2005; $17: 230-8$.

3. Probst AV, Almouzni G. Pericentric heterochromatin: dynamic organization during early development in mammals. Differentiation $2008 ; 76: 15-23$

4. Choo KH. Domain organization at the centromere and neocentromere. Dev Cell $2001 ; 1: 165-77$.

5. Pezer Z, Ugarkovic D. Role of non-coding RNA and heterochromatin in aneuploidy and cancer. Semin Cancer Biol 2008; 18 : 123-30.

6. Volpe TA, Kidner C, Hall IM, et al. Regulation of heterochromatic silencing and histone $\mathrm{H} 3$ lysine -9 methylation by RNAi. Science 2002 ; $297: 1833-7$

7. Verdel A, Jia S, Gerber $S$, et al. RNAi-mediated targeting of heterochromatin by the RITS complex. Science 2004 ; 303 : 672-6.

8. Folco HD, Pidoux AL, Urano T, et al. Heterochromatin and RNAi are required to establish CENP-A chromatin at centromeres. Science 2008 ; $319: 94-7$.

9. Chen ES, Zhang K, Nicolas $\varepsilon$, et al. Cell cycle control of centromeric repeat transcription and heterochromatin assembly. Nature 2008 ; 451: $734-7$.

10. Rudert F, Bronner S, Garnier JM, et al. Transcripts from opposite strands of gamma satellite DNA are differentially expressed during mouse development. Mamm Genome 1995; 6 : 76-83.

11. Lehnertz B, Ueda Y, Derijck AA, et al. Suv39h-mediated histone H3 lysine 9 methylation directs DNA methylation to major satellite repeats at pericentric heterochromatin. Curr Biol 2003; 13 : 1192-200.

12. Topp CN, Zhong CX, Dawe RK. Centromere-encoded RNAs are integral components of the maize kinetochore. Proc Natl Acad Sci USA 2004 ; $101: 15986-91$.

13. Fukagawa T, Nogami M, Yoshikawa M, et al. Dicer is essential for formation of the heterochromatin structure in vertebrate cells. Nat Cell Biol 2004 ; 6 : 784-91.

14. Maison C, Bailly D, Peters AH, et al. Higher-order structure in pericentric heterochromatin involves a distinct pattern of histone modification and an RNA component. Nat Genet $2002 ; 30: 329-34$.

15. Arney KL, Bao S, Bannister AJ, et al. Histone methylation defines epigenetic asymmetry in the mouse zygote. Int J Dev Biol 2002 ; $46: 317-20$.

16. Probst AV, Santos F, Reik W, et al. Structural differences in centromeric heterochromatin are spatially reconciled on fertilisation in the mouse zygote. Chromosoma 2007 ; $116: 403-15$.

17. Puschendorf M, Terranova R, Boutsma $\varepsilon$, et al. PRCl and Suv39h specify parental asymmetry at constitutive heterochromatin in early mouse embryos. Nat Genet $2008 ; 40: 411-20$. 
18. Peters AH, Kubicek S, Mechtler K, et al. Partitioning and plasticity of repressive histone methylation states in mammalian chromatin. Mol Cell $2003 ; 12: 1577-89$.

19. Manuelidis L. Indications of centromere movement during interphase and differentiation. Ann Ny Acad Sci 1985; $450: 205-21$

20. Alcobia I, Dilao R, Parreira L. Spatial associations of centromeres in the nuclei of hematopoietic cells: evidence for cell-type-specific organizational patterns. Blood 2000 ; 95 : 1608-15.

21. Terranova R, Sauer $S$, Merkenschlager $M$, et al. The reorganisation of constitutive heterochromatin in differentiating muscle requires HDAC activity. Exp Cell Res $2005 ; 310: 344-56$.

22. Baxter J, Sauer $S$, Peters $A$, et al. Histone hypomethylation is an indicator of epigenetic plasticity in quiescent lymphocytes. EMBO J 2004 ; $23: 4462-72$.

23. Grigoryev SA, Nikitina T, Pehrson JR, et al. Dynamic relocation of epigenetic chromatin markers reveals an active role of constitutive heterochromatin in the transition from proliferation to quiescence. $J$ Cell Sci $2004 ; 117: 6153-62$.

24. Martin C, Beaujean N, Brochard V, et al. Genome restructuring in mouse embryos during reprogramming and early development. Dev Biol 2006 ; $292: 317-32$.

25. Terranova R, Pereira CF, Du Roure C, et al. Acquisition and extinction of gene expression programs are separable events in heterokaryon reprogramming. J Cell Sci 2006 ; 119:2065-72.

26. Dernburg AF, Broman KW, Fung JC, et al. Perturbation of nuclear architecture by long-distance chromosome interactions. Cell 1996; $85: 745-59$.

27. Lanctot C, Cheutin T, Cremer M, et al. Dynamic genome architecture in the nuclear space: regulation of gene expression in three dimensions. Nat Rev Genet 2007 ; $8: 104-15$
28. Roldan $\varepsilon$, Fuxa M, Chong W, et al. Locus 'decontraction' and centromeric recruitment contribute to allelic exclusion of the immunoglobulin heavy-chain gene. Nat Immunol 2005 ; $6: 31-41$.

29. Grewal SI, Jia S. Heterochromatin revisited. Nat Rev Genet $2007 ; 8$ : 35-46.

30. Yuen KW, Montpetit B, Hieter P. The kinetochore and cancer: what's the connection? Curr Opin Cell Biol 2005; $17: 576-82$

31. Boveri. Zur Frage der Entstehung maligner Tumoren (The origin of malignant tumors). Jena: Gustav Fischer, 1914.

32. Weaver BA, Silk AD, Montagna $C$, et al. Aneuploidy acts both oncogenically and as a tumor suppressor. Cancer Cell $2007 ; 11: 25-36$.

33. Carroll CW, Straight AF. Centromere formation: from epigenetics to self-assembly. Trends Cell Biol $2006 ; 16: 70-8$.

34. Peters AH, O'Carroll D, Scherthan $\mathrm{H}$, et al. Loss of the Suv39h histone methyltransferases impairs mammalian heterochromatin and genome stability. Cell 2001; 107:323-37.

35. Taddei A, Maison C, Roche D, et al. Reversible disruption of pericentric heterochromatin and centromere function by inhibiting deacetylases. Nat Cell Biol $2001 ; 3: 114-20$.

36. Bouzinba-Segard $H$, Guais A, Francastel $C$. Accumulation of small murine minor satellite transcripts leads to impaired centromeric architecture and function. Proc Natl Acad Sci USA $2006 ; 103: 8709-14$

38. Verreault A. Hétérochromatine : un silence bien bruyant. Med Sci (Paris) 2003 ; 19 : 1181-2.

39. Nègre N, Cavalli G. Polycomb maîtrise la destinée cellulaire. Med Sci (Paris) 2006 ; $22: 1033-5$.

37. Labbé JP. Elasticité du centromère. Med Sci (Paris) $2005 ; 21: 261-6$.

40. Pâques F, Grange T. Architecture du noyau et régulation transcriptionnelle. Med Sci (Paris) $2002 ; 18: 1245-56$

41. Henckel A, Feil R. Asymétrie des génomes parentaux : implications en pathologie Med Sci (Paris) $2008 ; 24: 747-52$

\section{TIRÉS À PART}

R. Terranova

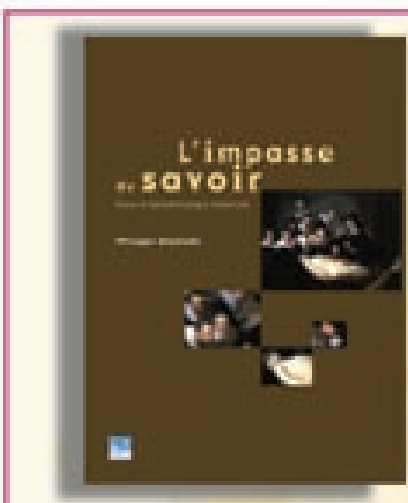

ISBN : 978-2-8425-4120-0 188 pages
L 'instauration des essais cliniques a été une révolution médicale de la seconde moitié du XXe siècle. En 2008, cette méthode d'acquisition du savoir s'essouffle. Les problématiques s'éloignent des préoccupations des cliniciens, l'applicabilité des résultats devient moins évidente, les recrutements se tarissent.

La nécessité d'un nouveau mode d'acquisition du savoir en médecine est la thèse ici développée par Philippe Abastado, cardiologue et docteur en épistémologie.

L'auteur s'adresse à l'honnête homme d'aujourd'hui intéressé par la médecine et les sciences humaines. Philippe Abastado, promoteur de l'épistémologie appliquée, a déjà publié «Cholestérol, maladie réelle et malade imaginaire » aux Empêcheurs de penser en rond, Seuil, 1999.

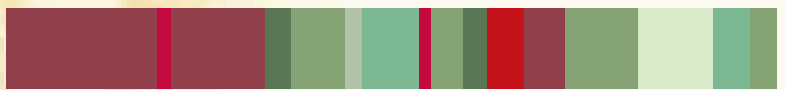

Philippe Abastado

À retourner à EDK, 2, rue Troyon - 92316 Sèvres Cedex

Tél. : 0155641393 - Fax : 0155641394 - E-mail : edk@edk.fr

NOM

Prónom

Adresse Adresse e-mail

Code postal :

TéL: :

Pays:

Fonction :

Je soutaite recenoir l'ouvrape

L'impasse du savoir : Prix public $18 €+3 €$ de port $=21 €$ TTC

7 Par chicque, a licedre de E D K

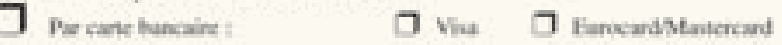

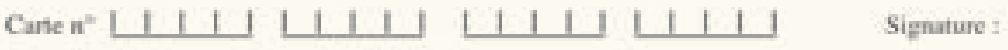

Dase d'expination :

$\mathrm{N}^{\circ}$ de contrôle au dos de la carte : 